\title{
Double-blind peer review
}

\author{
We are making author anonymity an option in our peer review.
}

Since Nature Nanotechnology launched in October 2006, our approach to peer review has been the same. We, like most science journals, use 'single-blind' peer review: that is, the referees of a paper know who the authors are but the authors don't know who the referees are. We are now making a change to this process and will offer 'doubleblind' peer review - in which referees review a paper without knowing who the authors are - as an option for authors submitting manuscripts to the journal. (Specific details will be available on our website once the option is activated.)

Our decision to offer double-blind has been driven by concerns from sections of our community that biases, such as those against female authors or researchers based at less prestigious labs and institutions, could play a role in the review process. The prevalence of these biases in the peerreview system is unclear ${ }^{1}$, but there is, for example, evidence of subtle bias against female students in other areas of academic life $^{2}$. Double-blind should, in theory, remove the possibility of such issues (be they unconscious or not), and surveys in various fields have found strong support for double-blind review ${ }^{3,4}$. We, therefore, think the option is worth exploring.

There are potential practical problems with double-blind peer review ${ }^{5}$, the most obvious being that, even without names on a paper, a referee can still guess the identity of the authors. Here though participating authors can help reduce the chances of identification by carefully wording their manuscripts, and our guide to authors will provide some suggestions for doing this. Authors interested in the option will also have to bear in mind that pre-publication discussion of results (be it at conferences or through preprint servers such as arXiv), which can be an important part of the research process, could also make it more difficult to remain anonymous during review.

Nature Nanotechnology is not the first Nature research journal to offer this option: double-blind peer review has been trialled at Nature Geoscience and Nature Climate Change since June 2013 $3^{6,7}$. During that time, the two journals have also been asking their authors to take part in a survey on the peer-review process. In a guest Thesis article in this issue ${ }^{8}$, Alastair Brown

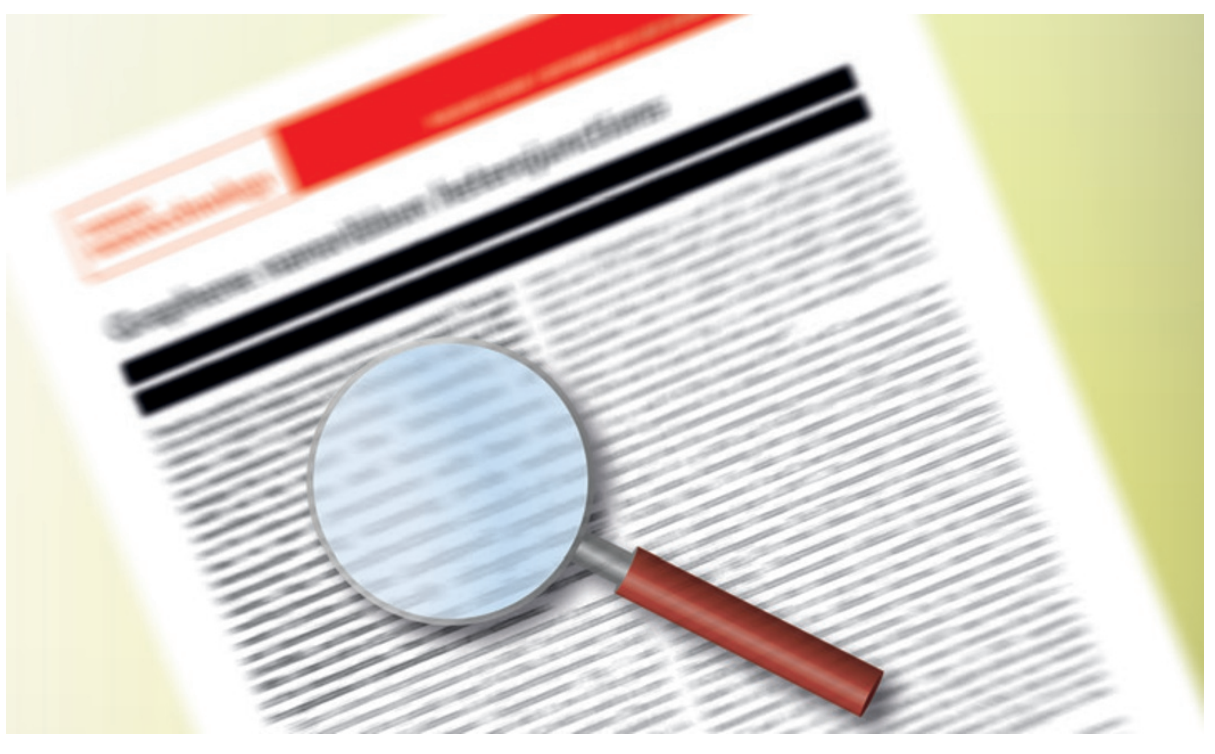

of Nature Climate Change discusses his experience of the double-blind process at the journal and the results of this survey so far. He notes, in particular, that although there is considerable support for doubleblind review in principle, the number of authors actually choosing the option at the two journals remains relatively low. Nature Nanotechnology covers a significant range of research fields and disciplines, and an analysis of our double-blind uptake could provide a valuable comparison between communities. We will also intend to carry out a survey on the peer-review process, which we hope our authors will take the time to complete. This should help us explore the ways in which we can improve our peer review.

\section{Surveys in various fields have found strong support for double-blind review.}

With the option of double-blind, we are of course adding an additional layer of secrecy to the peer-review process, and it could be argued that less would in fact be better. If referee reports were signed, and published along with the paper, referees would perhaps be just as likely to provide a balanced report as if the reports were anonymous 9 . At Nature Nanotechnology, we think that referee anonymity is still currently the most practical approach we have for securing the critical reports we need to make a decision on a work. (If a referee feels strongly about putting their name to their comments, we will though pass on a signed referee report to the authors.) Anonymity allows referees to comment freely and critically on a paper, unencumbered by thoughts of negative repercussions. Furthermore, it helps ensure that authors consider and respond to all referee reports based only on their content, and not on the name or standing of the referee themselves.

Our single-blind peer-review system has, we believe, served us well so far, and our decision to now offer double-blind review as an option at the journal has not been driven by a sense of dissatisfaction with our referees. But peer review is never perfect and we want to see if there are ways that we can make ours better.

References

1. Darling, E. Conserv. Biol. http://dx.doi.org/10.1111/cobi.12333 (2014)

2. Moss-Racusin, C. A., Dovidio, J. F., Brescoll, V. L., Graham, M. J. \& Handelsman, J. Proc. Natl Acad. Sci. USA 109, 16474-16479 (2012).

3. http://www.senseaboutscience.org/pages/peer-review.html

4. Nature Geosci. 5, 585 (2012).

5. Nature 451, 605-606 (2008).

6. Nature Geosci. 6, 413 (2013)

7. Nature Clim. Change 3, 525 (2013)

8. Brown, A. Nature Nanotech. 9, 871-872 (2014).

9. van Rooyen, S., Godlee, F., Evans, S., Black, N. \& Smith, R. BMJ 318, 23-27 (1999).

Published online: 2 November 2014 